\title{
EDITORIAL
}

\section{Open-angle glaucoma surgery: the current standing}

\author{
Cirurgia do glaucoma de ângulo aberto: estado atual
}

IVan Maynart TaVares

\begin{abstract}
Glaucoma is an optic neuropathy characterized by progressive degeneration of retinal ganglion cells ${ }^{(1)}$. It is the main cause of irreversible blindness in the world, and affects more than 70 million people worldwide, being $10 \%$ bilaterally blind ${ }^{(2)}$. Glaucoma can remain asymptomatic until it is severe, leading glaucoma filtration surgery to be performed in advanced cases, often by general ophthalmologists, which may be, in part, responsible for the wrong impression of its low success-rates and high incidence of complications. Therefore, glaucoma surgery is feared by patients and even by some ophthalmologists. Nevertheless, recently published studies on glaucoma surgery safety and efficacy have reported a different reality.

First, due to recent diagnostic and therapeutic advancements, including improvements in surgery techniques, the 20-year probability of glaucoma-related blindness in at least one eye was reduced to half during the period from 1965 to 2009(3). One of these enhancements was the advent of the safe trabeculectomy technique, proposed by Peng Khaw from the Moorfields Eye Hospital(4). Moreover, a multicenter analysis showed that good trabeculectomy outcomes with low rates of surgical complications can be achieved, but intensive proactive postoperative care is required. These authors showed an intraocular pressure (IOP) equal or less than $18 \mathrm{mmHg}$ in $86 \%$ of the patients after a two-year follow-up period, when included hypotensive medication use ${ }^{(5)}$. They also reported antifibrotics use in $93 \%$ of the cases, what corroborated the current practice pattern among glaucoma specialists. The use of mitomycin-C (MMC) during trabeculectomy has not only enhanced its IOP-lowering effect, but also made the postoperative period more challenging. Suture manipulation (including massage, laser suture lysis, etc.), bleb needling and subconjunctival 5-fluorouracil injections are directly related to success, and demand both time and expertise. In the same report, 31 patients (7\%) had late-onset hypotony (three of them with visual acuity decreased by $>2$ Snellen lines), and two patients developed bleb-related endophthalmitis ${ }^{(5)}$. Incidentally, another recent paper reported that visual loss after surgery, the "wipe-out" phenomenon, is rare, even in patients with advanced glaucomatous visual field loss and split fixation ${ }^{(6)}$.

The Ex-Press ${ }^{\circledR}$ implantation during glaucoma filtration surgery should be considered as a modification in trabeculectomy, and not as an entirely new procedure. A recent meta-analysis of randomized clinical trials comparing both procedures reported similar efficacy in IOP-lowering, medication reduction, vision recovery, qualified operative success-rates, and a greater incidence of most complications, with the exception of hyphema ${ }^{(7)}$. Controversy over its application in daily practice exists, as its efficacy and safety are similar to trabeculectomy, however it makes the procedure more costly.

Non-penetrating surgical procedures, including deep sclerectomy (DS), are more difficult to perform and require a longer learning curve. The addition of MMC to DS not only decreased the difference in the reduction in IOP (from -2.65 to $-0.83 \mathrm{mmHg}$ ), but also increased the incidence of complications, when compared to trabeculectomy with MMC. Even though, the absolute risk of complications is higher in the trabeculectomy group ${ }^{(8)}$. Hence, good result reports with this technique have been limited to a select group of skilled surgeons.

With regard to glaucoma drainage devices (GDD), the Tube versus Trabeculectomy Study (TVT), a multicenter, randomized clinical trial, demonstrated that IOP in the low-teens could be achieved with GDD $\left(350 \mathrm{~mm}^{2}\right.$ Baerveldt implant; Abbot Medical Optics Inc., USA), in patients with previous cataract extraction or trabeculectomy ${ }^{(9)}$. Although the TVT study has expanded the use of tube shunts beyond refractory glaucoma, a major shift in glaucoma surgical practice patterns has not been seen, and GDD continue to be often used either after two failed filtration surgeries or in cases in which these procedures are not indicated.

Cyclodestructive procedures, both trans-scleral and endoscopic, have been reserved for cases in which other surgical techniques have failed. Though they are effective, potential for serious complications exists ${ }^{(10)}$; therefore they are seldom indicated before filtration procedures or GDD.
\end{abstract}

Submitted for publication: June 30, 2014

Accepted for publication: June 30, 2014

Glaucoma Service, Department of Ophthalmology and Visual Sciences, Universidade Federal de São Paulo - Escola Paulista de Medicina - UNIFESP - São Paulo (SP) - Brazil.
Funding: No specific financial support was available for this study.

Disclosure of potential conflicts of interest: The author has any potential conflicts of interest to disclose. Corresponding author: Ivan Maynart Tavares. Rua Botucatu, 821 - São Paulo, SP - 04023-062 - Brazil E-mail: im.tavares@unifesp.br 
Finally, the advent of new devices and innovative techniques led on to new procedures, the minimally invasive glaucoma surgeries (MIGS). MIGS are ab interno and ab externo procedures, with limited manipulation of the sclera, little or no manipulation of the conjunctiva, and no bleb formation. Trabectome (NeoMedix, USA), Hydrus (Ivantis, USA), iStent (Glaukos, USA) and canaloplasty are some examples of MIGS approved in the USA or Europe. They are indicated for mild to moderate glaucoma, whereas IOP is reduced to mid-to-high teens, preferably combined with cataract extraction. Furthermore, most MIGS are fast to perform, have a more rapid visual recovery, and have a lower complication rate than filtration surgery ${ }^{(11,12)}$. Nonetheless, there is limited data on costs, as well as on how they could negatively affect a future trabeculectomy. Unfortunately, none of the MIGS devices is approved in Brazil, in part due to regulatory authorities costly and bureaucratic procedures and requirements for registration of medical devices, what have limited our armamentarium for glaucoma treatment and hampered science development.

\section{REFERENCES}

1. Weinreb RN, Aung T, Medeiros FA. The pathophysiology and treatment of glaucoma: a review. JAMA. 2014;311(18):1901-11.

2. Quigley HA, Broman AT. The number of people with glaucoma worldwide in 2010 and 2020. Br J Ophthalmol. 2006;90(3):262-7.

3. Malihi M, Moura Filho ER, Hodge DO, Sit AJ. Long-term trends in glaucoma-related blindness in Olmsted Country, Minnesota. Ophthalmology. 2014;121(1):134-41.

4. Khaw PT, Chiang M, Shah P, Sii F, Lockwood A, Khalil A. Enhanced trabeculectomy: the Moorfields Safer Surgery System. Dev Ophthalmol. 2012;50:1-28.

5. Kirwan JF, Lockwood AJ, Shah P, Macleod A, Broadway DC, King AJ, et al. Trabeculectomy in the $21^{\text {st }}$ century: a multicenter analysis. Ophthalmology. 2013;120(12):2532-9.

6. Balekudaru S, George R, Panday M, Singh M, Neog A, Lingam V. Prospective evaluation of early visual loss following glaucoma-filtering surgery in eyes with split fixation. J Glaucoma. 2014;23(4):211-8.
7. Wang W, Zhang X. Meta-Analysis of Randomized Controlled Trials Comparing EX-PRESS implantation with trabeculectomy for open-angle glaucoma. PLoS One. 2014;9(6):e100578.

8. Rulli E, Biagioli E, Riva I, et al. Efficacy and safety of trabeculectomy vs nonpenetrating surgical procedures: a systematic review and meta-analysis. JAMA Ophthalmol. 2013;131(12):1573-82.

9. Gedde SJ, Singh K, Schiffman JC, Feuer WJ. The Tube Versus Trabeculectomy Study: interpretation of results and application to clinical practice. Curr Opin Ophthalmol. 2012;23(2):118-26

10. Huang G, Lin SC. When should we give up filtration surgery: indications, techniques and results of cyclodestruction. Dev Ophthalmol. 2012;50:173-83.

11. Brandao LM, Grieshaber MC. Update on minimally invasive glaucoma surgery (MIGS) and new implants. J Ophthalmol. 2013:2013:705915.

12. Au L. Are newer surgical interventions for glaucoma making a difference? Br J Ophthalmol. 2014;98(1):1-2.

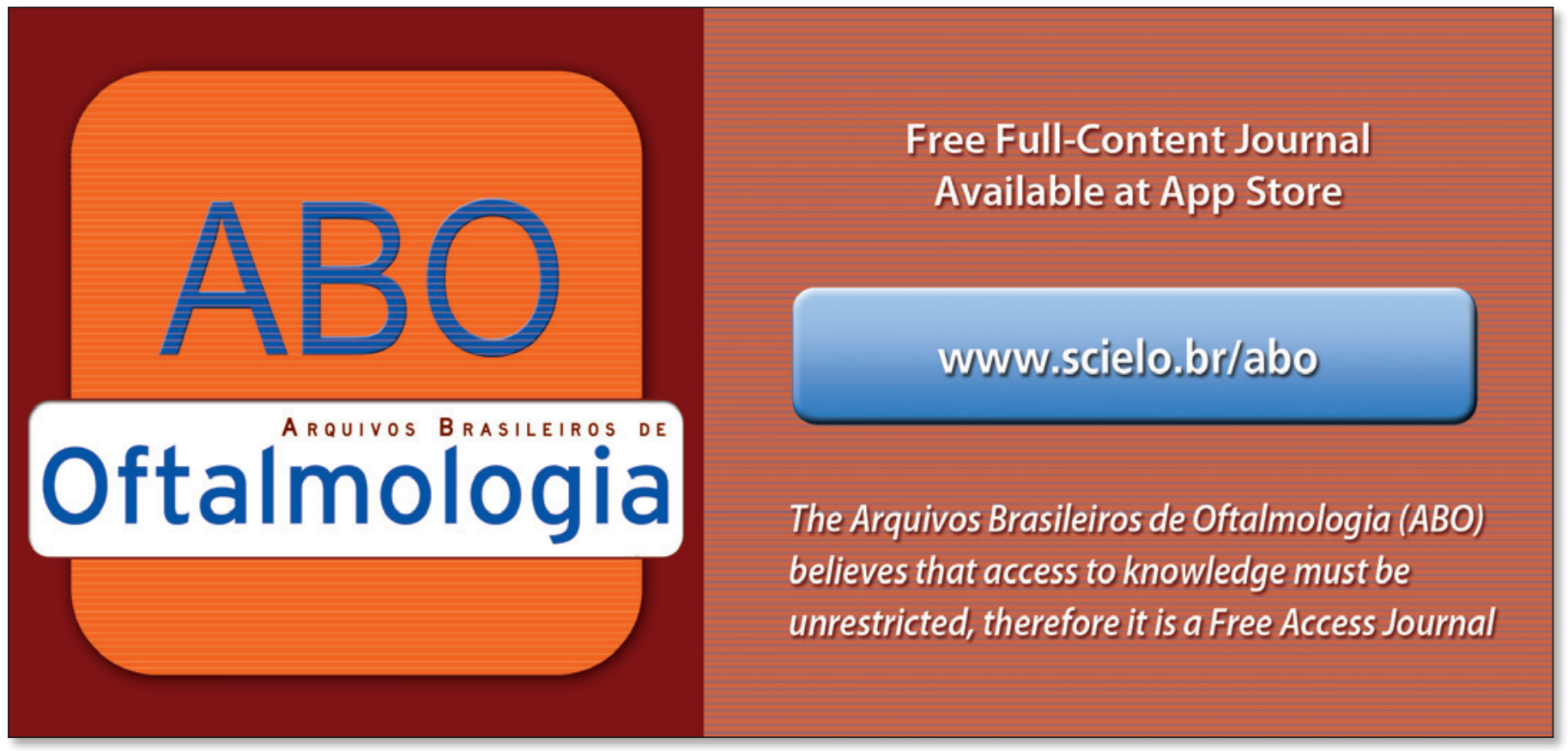

